\title{
Endoscopic third ventriculostomy and choroid plexus cauterization in infants with hydrocephalus: a retrospective Hydrocephalus Clinical Research Network study
}

\author{
Clinical article
}

\author{
Abhaya V. Kulkarni, M.D., Ph.D., ${ }^{1}$ Jay Riva-Cambrin, M.D., M.Sc., ${ }^{2}$ \\ Samuel R. Browd, M.D., Ph.D., ${ }^{3}$ James M. DraKe, M.B.B.Ch., M.Sc., ${ }^{1}$ \\ Richard HolubKov, Ph.D., ${ }^{2}$ John R. W. Kestle, M.D., M.Sc., ${ }^{2}$ \\ David D. Limbrick, M.D., Ph.D. ${ }^{4}$ Curtis J. Rozzelle, M.D., ${ }^{5}$ \\ Tamara D. Simon, M.D., M.S.P.H., ${ }^{3}$ Mandeep S. Tamber, M.D., Ph.D., ${ }^{6}$ \\ John C. Wellons III, M.D., M.S.P.H., ${ }^{7}$ and William E. Whitehead, M.D., M.Sc., ${ }^{8}$ \\ for the Hydrocephalus Clinical Research Network
}

\begin{abstract}
${ }^{1}$ Hospital for Sick Children, University of Toronto, Ontario, Canada $;{ }^{2}$ Primary Children's Medical Center, Salt Lake City, Utah; ${ }^{3}$ Seattle Children's Hospital, Seattle, Washington; ${ }^{4}$ St. Louis Children's Hospital, St. Louis, Missouri, ${ }^{5}$ Children's Hospital of Alabama, Birmingham, Alabama; ${ }^{6}$ Pittsburgh Children's Hospital, Pittsburgh, Pennsylvania; ${ }^{7}$ Vanderbilt University, Nashville, Tennessee; and ${ }^{8}$ Texas Children's Hospital, Houston, Texas
\end{abstract}

\begin{abstract}
Object. The use of endoscopic third ventriculostomy (ETV) with choroid plexus cauterization (CPC) has been advocated as an alternative to CSF shunting in infants with hydrocephalus. There are limited reports of this procedure in the North American population, however. The authors provide a retrospective review of the experience with combined ETV + CPC within the North American Hydrocephalus Clinical Research Network (HCRN).

Methods. All children ( $<2$ years old) who underwent an ETV + CPC at one of 7 HCRN centers before November 2012 were included. Data were collected retrospectively through review of hospital records and the HCRN registry. Comparisons were made to a contemporaneous cohort of 758 children who received their first shunt at $<2$ years of age within the HCRN.

Results. Thirty-six patients with ETV + CPC were included (13 with previous shunt). The etiologies of hydrocephalus were as follows: intraventricular hemorrhage of prematurity (9 patients), aqueductal stenosis (8), myelomeningocele (4), and other (15). There were no major intraoperative or early postoperative complications. There were 2 postoperative CSF infections. There were 2 deaths unrelated to hydrocephalus and 1 death from seizure. In 18 patients ETV + CPC failed at a median time of 30 days after surgery (range 4-484 days). The actuarial 3-, 6-, and 12 -month success for ETV + CPC was 58\%, 52\%, and 52\%. Time to treatment failure was slightly worse for the 36 patients with ETV + CPC compared with the 758 infants treated with shunts $(\mathrm{p}=0.012)$. Near-complete CPC $(\geq 90 \%)$ was achieved in 11 cases (31\%) overall, but in 50\% (10 of 20 cases) in 2012 versus 6\% ( 1 of 16 cases) before 2012 (p $=0.009)$. Failure was higher in children with $<90 \%$ CPC $(\mathrm{HR} 4.39,95 \%$ CI 0.999-19.2, $\mathrm{p}=0.0501)$.

Conclusions. The early North American multicenter experience with ETV + CPC in infants demonstrates that the procedure has reasonable safety in selected cases. The degree of CPC achieved might be associated with a surgeon's learning curve and appears to affect success, suggesting that surgeon training might improve results. (http://thejns.org/doi/abs/10.3171/2014.6.PEDS13492)
\end{abstract}

KEY WORDS • hydrocephalus • endoscopy • choroid plexus • shunt

$\mathrm{S}$ HUNTING remains the most common treatment for hydrocephalus, but is associated with several shortand long-term complications. Endoscopic third ven-

Abbreviations used in this paper: CCHU = CURE Children's Hospital of Uganda; CPC = choroid plexus cauterization; ETV = endoscopic third ventriculostomy; ETVSS = ETV Success Score; FIESTA/CISS = fast imaging employing steady-state acquisition/ constructive interference in steady state; HCRN = Hydrocephalus Clinical Research Network; IVH = intraventricular hemorrhage. triculostomy (ETV) is the only alternative to shunting for hydrocephalus. There are many patients, however, whose success rate with ETV is very low. ${ }^{1,2,5,8}$ Limited experience with the use of endoscopic choroid plexus cauterization (CPC) alone (without ETV) was reported by Scarff in $1952^{13}$ and Pople and Ettles in 1995..$^{2}$ More recently,

This article contains some figures that are displayed in color online but in black-and-white in the print edition. 
however, the use of ETV combined with CPC has been advocated as a means of increasing the overall treatment success and avoiding the need for a shunt. When studied in a large sub-Saharan African population, the use of CPC substantially improved the chance of ETV success, with a dose-response effect (partial CPC was approximately half as effective as full CPC) ${ }^{15,16,18}$ Based on this, there has been increasing interest in the use of combined ETV + CPC in the North American hydrocephalus population. Warf et al. have reported their early experience with 10 infants in whom IVH of prematurity was treated with ETV + CPC. ${ }^{17}$ The procedure failed in a total of 6 , all of whom had demonstrated prepontine cisternal scarring at surgery and on preoperative fast imaging employing steady-state acquisition/constructive interference in steady state (FIESTA/CISS) MRI studies.

When this report was prepared, the Hydrocephalus Clinical Research Network (HCRN) comprised 7 pediatric neurosurgery centers dedicated to the study of children with hydrocephalus. As a first step in investigating the efficacy of ETV + CPC, we retrospectively reviewed the outcome of ETV + CPC procedures that had been performed at the HCRN centers. The objective of this study was to determine the morbidity, complication incidence, and efficacy of ETV + CPC at major pediatric neurosurgery centers in North America. Because the primary interest in ETV + CPC has been its possible efficacy in infants, we limited our focus to patients who were $<2$ years of age when ETV + CPC was performed.

\section{Patient Population}

\section{Methods}

The study population consisted of children $<2$ years old, with new-onset hydrocephalus or those with previous CSF shunts or ETV who underwent an ETV + CPC procedure at one of 7 HCRN centers (Children's Hospital of Alabama, Birmingham, AL; Primary Children's Hospital, Salt Lake City, UT; Seattle Children's Hospital, WA; Children's Hospital of Pittsburgh, PA; St. Louis Children's Hospital, MO; Texas Children's Hospital, Houston, TX; Sick Kids Hospital, Toronto, Canada) between January 1, 2000, and November 1, 2012. The cases were identified by review of local operative databases at each center and by review of the HCRN Core Data Project registry, which prospectively tracked all hydrocephalus surgeries at each HCRN center beginning as early as April 2008 in some centers, with staggered start-up thereafter. Data were collected retrospectively through review of hospital medical charts, imaging records, and data in the HCRN registry.

Data from HCRN Core Data Project registry for patients who had received a CSF shunt were used to compare time to treatment failure. This group consisted of 758 patients who were $<2$ years of age at the time of first shunt placement.

\section{Perioperative Data}

We recorded patient age at ETV + CPC, etiology of hydrocephalus, previous treatment of hydrocephalus, duration of surgery, significant intraoperative complications (including severe bleeding or anatomical brain injury), and the estimated amount of the choroid plexus within both lateral ventricles that was successfully cauterized (categorized as none, $<40 \%, 40 \%$ to $<60 \%, 60 \%$ to $<$ $90 \%$, or $\geq 90 \%$ ). These data, including the amount of choroid plexus cauterized, were based on review of the operative notes.

\section{Imaging Data}

Preoperative imaging was reviewed to determine ventricle size based on the frontal/occipital horn ratio, $, 7,11$ the third ventricle morphology index,${ }^{4}$ and the presence of prepontine adhesions on FIESTA/CISS MRI studies, if available.

\section{Outcome Measures}

The primary outcome was the time to the occurrence of ETV + CPC failure (recorded from date of surgery), which was defined as follows: recurrence of symptomatic hydrocephalus from failure of ETV + CPC and/or the presence of loculated compartments, requiring repeat CSF diversion surgery, or death. We also recorded the presence of CSF infection, and of intraoperative, perioperative, and delayed postoperative complications, including any new neurological deficits.

\section{Data Analysis}

The primary analysis was descriptive; we determined the time to first treatment failure after ETV + CPC by using the Kaplan-Meier survival method. This was compared with time-to-failure data for patients $<2$ years old who had received CSF shunting; these data were obtained from the HCRN Core Data Project registry. Patients in this cohort represented all children with first-time CSF shunt insertions who were $<24$ months of age at the time of shunt surgery.

Univariate Cox regression was performed to determine the impact of the following independent variables on time to first failure of ETV + CPC: patient age (months); etiology of hydrocephalus (categorized as IVH of prematurity, aqueductal stenosis, myelomeningocele, or other); amount of CPC actually performed (which was analyzed in the 4 categories as collected, as well as dichotomized as $\geq 90 \%$ versus $<90 \%$ ); and calendar year in which the procedure was performed (2012 vs before 2012).

We also calculated the ETV Success Score ([ETVSS $]^{8}$ - a validated ${ }^{3,9,10,14}$ score developed in a primarily North American and European sample that predicts success of ETV) for each patient, and their CURE Children's Hospital of Uganda (CCHU) ETVSS ${ }^{18}$ (a score developed in a sub-Saharan population that predicts success of ETV and accounts for the effects of CPC). The ETVSS and CCHU ETVSS were also tested in separate univariate analyses to determine if they were significantly associated with success of ETV + CPC in our cohort.

\section{Results}

A total of 43 patients (age range 4 days to 19 years) were treated with ETV + CPC. Of these, 36 were $<2$ years old at surgery and formed the basis of further analysis. The 
$\mathrm{ETV}+\mathrm{CPC}$ procedure was performed as early as 2006 , but more frequently since 2011 within the HCRN (Fig. 1). A total of 12 surgeons performed the 36 procedures, and the median number of cases per surgeon was 3 (range 1-6). Baseline patient characteristics are shown in Table 1.

Eleven children (31\%), of whom 1 had evidence of prepontine scarring, had preoperative FIESTA/CISS MRI studies.

\section{Surgical Details}

Surgical details are shown in Table 2. In 12 patients either a reservoir (5) or external ventricular drain (7) was placed at the time of surgery. The ETV + CPC technique described by Warf et al. ${ }^{15}$ explicitly requires the use of a flexible endoscope. The majority of cases (28 [78\%]) in our cohort, however, used various rigid endoscopes as opposed to the flexible endoscope (8 [22\%]). When the flexible scope was used, cauterization of $\geq 90 \%$ of the choroid plexus was performed in $88 \%$ of cases (7 of 8) versus $14 \%$ of cases ( 4 of 28 ) when the rigid scope was used ( $\mathrm{p}<0.001$, Fisher exact test).

Cauterization of $\geq 90 \%$ of the choroid plexus was performed in $10(50 \%)$ of 20 cases in 2012 and in $1(6 \%)$ of 16 cases before 2012 ( $p=0.009$, Fisher exact test). The median duration of surgery was 116 minutes in 2012 and 80 minutes before 2012 ( $\mathrm{p}=0.31$, Wilcoxon test).

No major intraoperative or early postoperative complications were noted. Minor forniceal injury and a thalamic contusion were noted in 1 child each. Intraoperative bleeding was noted in 14 patients (39\%), but none of these episodes were severe (Table 2). Postoperative CSF infection was documented in 2 children (6\%), 1 of whom had an external ventricular drain placed at surgery. No significant neurological deficits were documented.

\section{Postoperative Outcome}

In a total of 18 patients (50\%), ETV + CPC failed at a median time of 30 days after surgery (range 4 days to
484 days). Two deaths were reported among the patients in whom this treatment failed. One child with procedure failure at 6 days after ETV + CPC died approximately 1.5 years later of abdominal compartment syndrome. Another child, who had a history of previous ventriculitis and a preexisting seizure disorder, experienced procedure failure 26 days after ETV + CPC. This child was treated with a shunt, and died approximately 10 months later of seizure. Among the patients who did not have documented failure, the median duration of follow-up was 10 months. One child in whom the procedure did not fail died of pneumonia 75 days after ETV + CPC, while receiving palliative care.

Univariate Cox analysis of time to treatment failure revealed no significant difference in failure of ETV + CPC based on age in days $(\mathrm{p}=0.92)$; etiology (categorized as aqueductal stenosis, myelomeningocele, IVH of prematurity, or other $[\mathrm{p}=0.84]$ ); completeness of CPC (percentage of choroid plexus cauterized) as analyzed in the original 4 categories $(p=0.17)$; ETVSS $(p=0.48)$; or CCHU ETVSS $(\mathrm{p}=0.28)$. However, failure occurred in $16(64 \%)$ of 25 children in whom $<90 \%$ of the choroid plexus was cauterized, compared with $2(18 \%)$ of 11 children with $\geq 90 \%$ CPC achieved, and this dichotomous comparison of failure rates was nearly significant according to the Cox model (hazard ratio 4.39 for failure if $<90 \%$ CPC achieved vs if $\geq 90 \%$ achieved, 95\% CI $0.999-19.2, \mathrm{p}=0.0501)$.

We collected a comparison cohort of all 758 children from the HCRN Core Data Project registry who were < 2 years of age at first shunt surgery. Their demographic details are shown in Table 1 . The distribution of age $(\mathrm{p}=$ 0.0016 according to the Mantel-Haenszel chi-square test) and etiology $(\mathrm{p}=0.0025$, chi-square test $)$ differed between the 2 cohorts. The Kaplan-Meier survival curve for time to treatment failure, comparing the ETV + CPC cohort and the shunt cohort, is shown in Fig. 2. The 3-month, 6-month, and 1-year success for ETV + CPC versus shunt

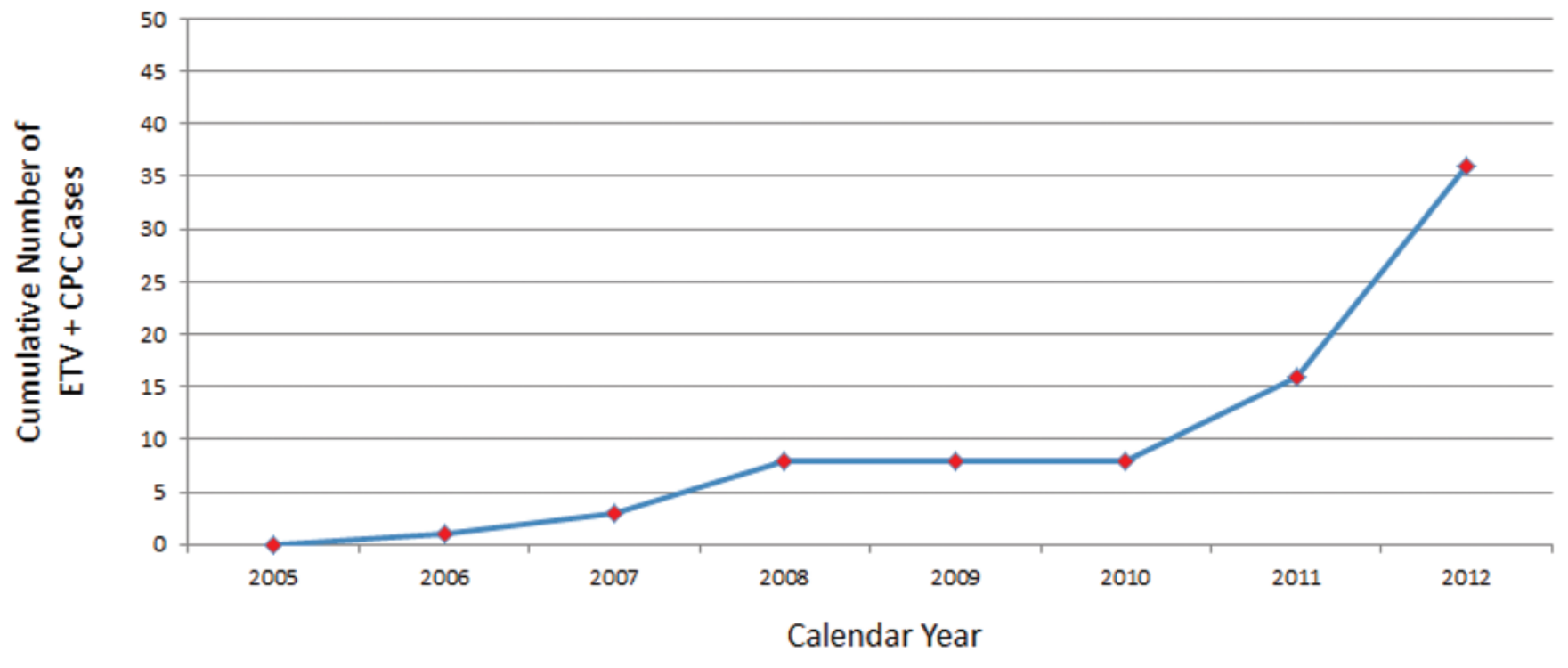

FIG. 1. Graph showing the cumulative number of patients in whom ETV + CPC procedures were performed within the HCRN. 
TABLE 1: Baseline characteristics in patients with hydrocephalus who underwent either ETV + CPC or shunt placement*

\begin{tabular}{lcc}
\hline \multicolumn{1}{c}{ Characteristic } & ETV + CPC Cohort & Shunt Cohort \\
\hline $\begin{array}{l}\text { total no. of patients } \\
\text { etiology }\end{array}$ & 36 & 758 \\
IVH of prematurity & $9(25)$ & $223(29)$ \\
aqueductal stenosis & $8(22)$ & $76(10)$ \\
myelomeningocele & $4(11)$ & $163(22)$ \\
congenital communicating & $2(6)$ & $65(9)$ \\
$\quad$ hydrocephalus & & \\
head injury & $2(6)$ & $17(2)$ \\
postinfectious & $2(6)$ & $29(4)$ \\
other† & $9(25)$ & $185(24)$ \\
age at ETV + CPC surgery & & \\
$<2$ mos & $9(25)$ & $316(42)$ \\
2 to <6 mos & $11(31)$ & $261(34)$ \\
6 to <12 mos & $10(28)$ & $110(15)$ \\
12 mos to 2 yrs & $6(17)$ & $71(9)$ \\
previous CSF shunt & $13(36)$ & 0 \\
median preop FOR (range) & $0.59(0.35-0.87)$ & $\mathrm{NA}$ \\
median preop TVMI (range) & $0.43(0.09-3.14)$ & $\mathrm{NA}$ \\
median ETVSS (range) & $50(10-80)$ & $46(10-80)$ \\
median CCHU ETVSS (range) & $4(2-8)$ & $1(0-5)$ \\
\hline
\end{tabular}

* Unless otherwise indicated, values in tables are expressed as the number (\%). FOR = frontal-occipital horn ratio; NA = not available; TVMI $=$ third ventricle morphology index.

† Category includes craniosynostosis, Dandy-Walker, tectal glioma, arachnoid cyst, intraparenchymal brain hemorrhage, and other etiologies.

was $58 \%$ versus $79 \%, 52 \%$ versus $72 \%$, and $52 \%$ versus $65 \%$, respectively. There was a statistically significant difference in time to treatment failure between the ETV + CPC group and the CSF shunt group $(p=0.012$, log-rank test). When adjusted for ETVSS (which accounts for the

TABLE 2: Operative and postoperative results in 36 patients with hydrocephalus who underwent ETV + CPC

\begin{tabular}{ll}
\hline \multicolumn{1}{c}{ Result } & Value \\
\hline median duration of surgery in min (range) & $109(55-191)$ \\
amount of choroid plexus cauterized & \\
$<40 \%$ & $6(17)$ \\
$40 \%$ to $<60 \%$ & $6(17)$ \\
$60 \%$ to $<90 \%$ & $13(36)$ \\
$\geq 90 \%$ & $11(31)$ \\
bleeding during surgery & \\
none & $22(61)$ \\
mild & $13(36)$ \\
moderate & $1(3)$ \\
severe & 0 \\
median postop FOR (range) & $0.55(0.38-0.93)$ \\
\hline
\end{tabular}

prognostic effects of age and etiology) in a Cox regression model, the results were essentially unchanged.

Data for infants who had undergone ETV alone without CPC were not available from the HCRN cohort. However, we estimated the predicted chance of ETVonly success at 6 months based on the mean ETVSS for the study cohort. For all 36 patients, the mean predicted chance of ETV-only success was $50 \%$ compared with the observed success of $52 \%$. For those in whom $<90 \%$ CPC was achieved, the mean chance of ETV-only success was $50 \%$ compared with observed success of $36 \%$. However, for those in whom $\geq 90 \%$ CPC was achieved, the mean chance of ETV-only success was $51 \%$ compared with the observed success of $82 \%$.

\section{Discussion}

We present, to our knowledge, the first multicenter retrospective experience with ETV + CPC in North America. Our objective was to establish the preliminary evidence of safety and efficacy of this new procedure in North American centers. It is important to note that in all cases this represents an early surgeon experience, without formal mentored training in the technique. Patients were selected on a purely ad hoc basis by the treating surgeon and came from a broad mix of etiologies. It is likely that several were patients undergoing salvage procedures, in whom this treatment was a last resort in the setting of difficult CSF management. Also, the technique used varied among the surgeons and often did not adhere to the description of Warf et al., and specifically to Warf's recommendation to use the flexible endoscope..$^{15}$

With this as the background, we believe our results suggest that ETV + CPC is, at least, a reasonably safe procedure. Among the deaths, there was only 1 neurological death from seizure; this occurred in a patient with a preexisting seizure disorder in whom ETV + CPC had already failed and who had received a shunt. The CSF infection rate was $6 \%$, and no significant neurological deficits or severe intraoperative bleeding were encountered.

In terms of procedure efficacy, the HCRN was uniquely able to provide a relatively contemporaneous cohort of infants with shunts against whom we could compare treatment failure outcomes. The survival rate for ETV + CPC was found to be lower than in the comparison shunt cohort. Although we did not have a comparable cohort of ETV-only infants, the observed success of the entire ETV + CPC cohort was no different than the predicted chance of ETV-only success based on the mean ETVSS for the study sample. However, the success was better in those in whom $\geq 90 \%$ CPC was achieved (82\%), and this compared favorably to the 6-month success of the shunt cohort $(72 \%)$ and the predicted chance of ETVonly success $(51 \%)$.

Identifying determinants of treatment success was limited by our small sample size. Nevertheless, near-complete CPC was nearly significant in its association with better success $(p=0.0501)$. Our study is one of the first to independently confirm the importance of near-complete CPC outside of the work of Warf's group. ${ }^{18}$ Our results further suggest that the degree of cauterization might be subject 
A. V. Kulkarni et al.

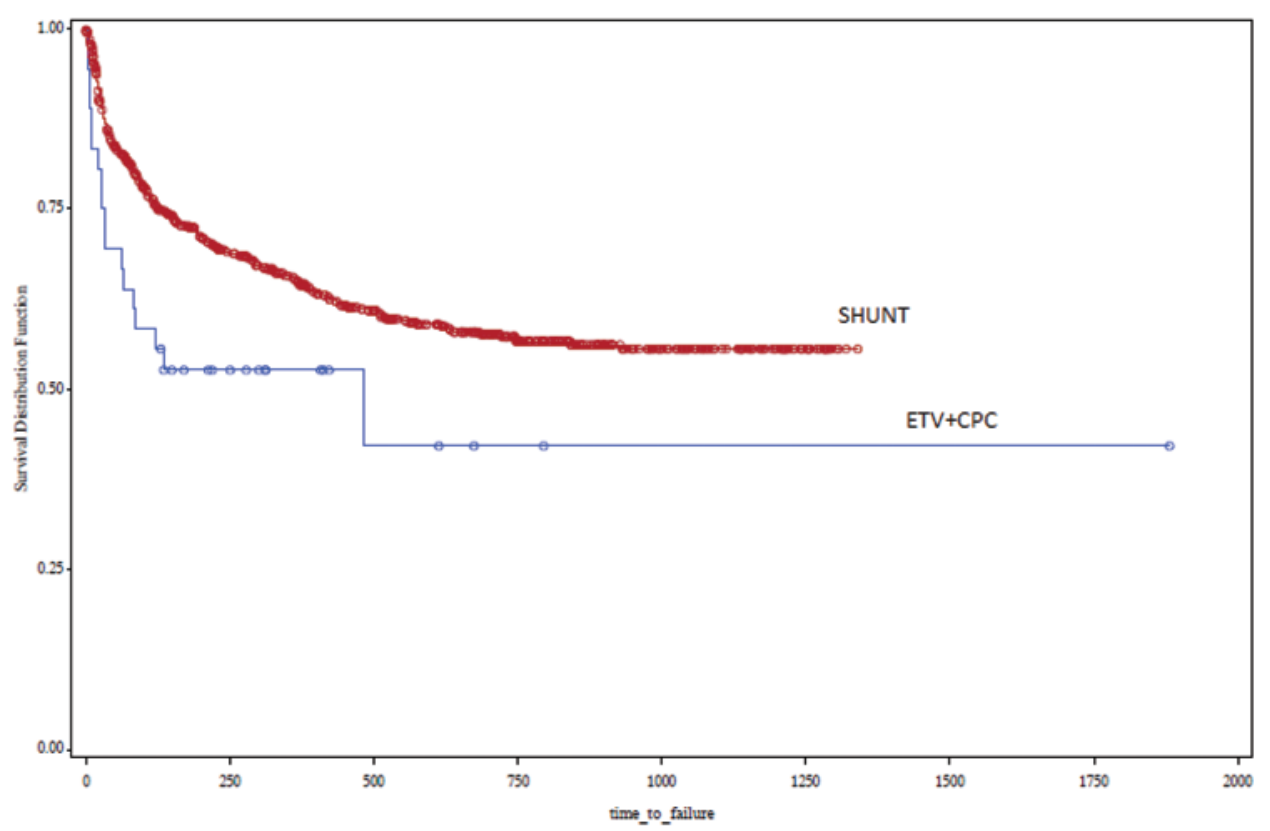

FIG. 2. Kaplan-Meier survival curve of time to failure of the procedure in the ETV + CPC cohort compared with a contemporaneous shunt cohort from the HCRN. Values on the $x$ axis represent days.

to a technical learning curve, because near-complete CPC was achieved in $50 \%$ of cases performed in 2012 compared with only $6 \%$ of earlier cases $(\mathrm{p}=0.009)$. Degree of CPC also seemed to be related to comfort in the use of the flexible scope rather than the rigid one $(\mathrm{p}<0.001)$. Our series was meant to provide results for the very early experience with this procedure, and, because of initial lack of comfort with the flexible scope by some surgeons, the rigid scope was often used. Although this does not follow the procedure as described by Warf et al., ${ }^{15}$ it provides a realistic picture of how many are initially attempting to do this operation. This provided us a unique opportunity to examine the effects of a rigid versus a flexible scope, and we showed that there is benefit to the flexible scope and to performing the procedure as described by Warf et al. We believe that this is an important message to surgeons who might be attempting this procedure for the first time and who often do choose to use the rigid scope. None of the surgeons who performed ETV + CPC in our study had received formal mentored training in the procedure at the time of analysis of this study. It is possible that with formal training, results of this procedure might be improved.

\section{Limitations of the Study}

The retrospective nature of this study poses several methodological limitations. First, we were only able to identify patients in whom CPC was at least performed to some degree. We could not retrospectively identify all cases in which the surgeon had intended to perform CPC and then abandoned the procedure. In a prospective intention-to-treat study, such cases would need to be included and would probably result in a higher reported failure rate. Second, the degree of CPC performed and the presence of intraoperative complications and bleeding was based on review of operative notes and was clearly subject to some inaccuracy. Third, patient selection and, importantly, pro- cedure failure were both determined only by the treating surgeon, with no independent adjudication. Varying individual thresholds for determining both the need for operative hydrocephalus intervention and repeat intervention could artificially impact observed treatment failure rates, independent of the actual efficacy of the procedure itself. Fourth, because many of the cases were performed fairly recently, the length of follow-up is relatively short. Finally, our small sample size limited our ability to fully explore secondary analyses of determinants of treatment success. Taken together, these limitations force us to be very cautious in the interpretation of our results and emphasize the preliminary and exploratory nature of our study.

\section{Future Work}

Having established early evidence of at least procedure safety, our Network is planning a prospective study of ETV + CPC that will address the limitations of the current study. This prospective study will involve a priori patient selection criteria, independent adjudication of outcome, and standardized surgeon training in the procedure at established centers. Since the current study was completed, several HCRN surgeons have invested in formal training, including travel to CURE Children's Hospital of Uganda, where this procedure was popularized and whose surgeons have the largest experience in its performance. We anticipate that this formal training will help in the early learning curve of this procedure and provide the best possible setting to test the efficacy of ETV + CPC prospectively in North America.

\section{Conclusions}

Our retrospective, multicenter North American study of the early experience with ETV + CPC suggests that 
it is a relatively safe procedure. Greater technical proficiency might improve treatment success, but this might be subject to a learning curve. Further careful prospective study of ETV + CPC in North America is warranted and necessary.

\section{Acknowledgments}

We thank our colleagues who kindly agreed to participate in this HCRN project and allow data collection for their patients for the purpose of this publication: Richard Ellenbogen, Jeffery Ojemann, Amy Lee, Douglas Brockmeyer, Peter Dirks, James Rutka, Michael Taylor, Leslie Ackacpo-Satchivi, Jeff Blount, Daniel Curry, Robert Dauser, Andrew Jea, Ian Pollack, Stephanie Greene, Elizabeth Tyler-Kabara, T.S. Park, Jeffrey Leonard, and Matthew Smyth. In addition, this work would not have been possible without the outstanding support of the team of dedicated personnel at each clinical site and the data coordinating center. We thank them for their hard work for the HCRN.

\section{Disclosure}

Dr. Browd has an ownership interest in Aqueduct Neurosciences, Inc., and in Navisonics, Inc.

The HCRN has been funded by private philanthropy and National Institute of Neurological Disorders and Stroke (NINDS) Grant No. 1RC1NS068943-01. Dr. Simon is supported by Award K23NS062900 from the NINDS, the Children's Hospital Association via the Pediatric Research in Inpatient Setting Network Executive Council, and Seattle Children's Center for Clinical and Translational Research. None of the sponsors participated in design and conduct of the study; collection, management, analysis, and interpretation of the data; or preparation, review, or approval of the manuscript. Its contents are solely the responsibility of the authors and do not necessarily represent the official view of the sponsors.

Author contributions to the study and manuscript preparation include the following. Conception and design: Kulkarni, RivaCambrin, Drake, Kestle, Limbrick, Rozzelle, Tamber, Wellons, Whitehead. Acquisition of data: Kulkarni, Riva-Cambrin, Browd, Limbrick, Rozzelle, Tamber, Wellons, Whitehead. Analysis and interpretation of data: Kulkarni, Riva-Cambrin, Holubkov, Kestle, Limbrick, Simon, Tamber, Wellons, Whitehead. Drafting the article: Kulkarni. Critically revising the article: all authors. Reviewed submitted version of manuscript: all authors. Approved the final version of the manuscript on behalf of all authors: Kulkarni. Statistical analysis: Kulkarni, Riva-Cambrin, Holubkov. Study supervision: Kulkarni, Kestle.

\section{References}

1. Buxton N, Macarthur D, Mallucci C, Punt J, Vloeberghs M: Neuroendoscopic third ventriculostomy in patients less than 1 year old. Pediatr Neurosurg 29:73-76, 1998

2. Drake JM: Endoscopic third ventriculostomy in pediatric patients: the Canadian experience. Neurosurgery 60:881-886, 2007

3. Durnford AJ, Kirkham FJ, Mathad N, Sparrow OC: Endoscopic third ventriculostomy in the treatment of childhood hydrocephalus: validation of a success score that predicts long-term outcome. Clinical article. J Neurosurg Pediatr 8:489-493, 2011

4. Foroughi M, Wong A, Steinbok P, Singhal A, Sargent MA, Cochrane DD: Third ventricular shape: a predictor of endoscopic third ventriculostomy success in pediatric patients. Clinical article. J Neurosurg Pediatr 7:389-396, 2011

5. Kadrian D, van Gelder J, Florida D, Jones R, Vonau M, Teo C, et al: Long-term reliability of endoscopic third ventriculostomy. Neurosurgery 62 (Suppl 2):614-621, 2008
6. Kulkarni AV, Drake JM, Armstrong DC, Dirks PB: Imaging correlates of successful endoscopic third ventriculostomy. J Neurosurg 92:915-919, 2000

7. Kulkarni AV, Drake JM, Armstrong DC, Dirks PB: Measurement of ventricular size: reliability of the frontal and occipital horn ratio compared to subjective assessment. Pediatr Neurosurg 31:65-70, 1999

8. Kulkarni AV, Drake JM, Mallucci CL, Sgouros S, Roth J, Constantini S: Endoscopic third ventriculostomy in the treatment of childhood hydrocephalus. J Pediatr 155:254-259.e1, 2009

9. Kulkarni AV, Riva-Cambrin J, Browd SR: Use of the ETV Success Score to explain the variation in reported endoscopic third ventriculostomy success rates among published case series of childhood hydrocephalus. Clinical article. J Neurosurg Pediatr 7:143-146, 2011

10. Naftel RP, Reed GT, Kulkarni AV, Wellons JC: Evaluating the Children's Hospital of Alabama endoscopic third ventriculostomy experience using the Endoscopic Third Ventriculostomy Success Score: an external validation study. Clinical article. J Neurosurg Pediatr 8:494-501, 2011

11. O’Hayon BB, Drake JM, Ossip MG, Tuli S, Clarke M: Frontal and occipital horn ratio: a linear estimate of ventricular size for multiple imaging modalities in pediatric hydrocephalus. Pediatr Neurosurg 29:245-249, 1998

12. Pople IK, Ettles D: The role of endoscopic choroid plexus coagulation in the management of hydrocephalus. Neurosurgery 36:698-702, 1995

13. Scarff JE: Nonobstructive hydrocephalus; treatment by endoscopic cauterization of the choroid plexus. Long term results. J Neurosurg 9:164-176, 1952

14. Vogel TW, Bahuleyan B, Robinson S, Cohen AR: The role of endoscopic third ventriculostomy in the treatment of hydrocephalus. Clinical article. J Neurosurg Pediatr 12:54-61, 2013

15. Warf BC: Comparison of endoscopic third ventriculostomy alone and combined with choroid plexus cauterization in infants younger than 1 year of age: a prospective study in 550 African children. J Neurosurg 103 (6 Suppl):475-481, 2005

16. Warf BC, Campbell JW: Combined endoscopic third ventriculostomy and choroid plexus cauterization as primary treatment of hydrocephalus for infants with myelomeningocele: long-term results of a prospective intent-to-treat study in 115 East African infants. Clinical article. J Neurosurg Pediatr 2:310-316, 2008

17. Warf BC, Campbell JW, Riddle E: Initial experience with combined endoscopic third ventriculostomy and choroid plexus cauterization for post-hemorrhagic hydrocephalus of prematurity: the importance of prepontine cistern status and the predictive value of FIESTA MRI imaging. Childs Nerv Syst 27:1063-1071, 2011

18. Warf BC, Mugamba J, Kulkarni AV: Endoscopic third ventriculostomy in the treatment of childhood hydrocephalus in Uganda: report of a scoring system that predicts success. Clinical article. J Neurosurg Pediatr 5:143-148, 2010

Manuscript submitted September 24, 2013.

Accepted June 2, 2014.

Please include this information when citing this paper: published online July 4, 2014; DOI: 10.3171/2014.6.PEDS13492.

Address correspondence to: Abhaya V. Kulkarni, M.D., Ph.D., F.R.C.S.C., Division of Neurosurgery, Hospital for Sick Children, Rm. 1503, 555 University Ave., Toronto, ON M5G 1X8, Canada. email: abhaya.kulkarni@sickkids.ca. 